\title{
Optimal regulatory control of early contract
} termination

Evatt, G.W and Johnson, P and Duck, P

2013

MIMS EPrint: 2013.30

Manchester Institute for Mathematical Sciences

School of Mathematics

The University of Manchester

\footnotetext{
Reports available from: http://eprints.maths.manchester.ac.uk/

And by contacting: The MIMS Secretary

School of Mathematics

The University of Manchester

Manchester, M13 9PL, UK
} 
IMA Journal of Management Mathematics Page 1 of 17

doi:10.1093/imaman/dpnxxx

\title{
Optimal regulatory control of early contract termination
}

\author{
G.W. EvatT, P.V. Johnson, \& P.W. Duck \\ School of Mathematics, Alan Turing Building, University of Manchester, \\ Oxford Road, Manchester, M13 9PL, UK.
}

[Received on X October 2012]

\begin{abstract}
We present a quantitative method to find jointly optimal strategies for an industry regulator and a firm, who operate under exogenous uncertainty. The firm controls its operating policy in order to maximize its expected future profits, whilst taking account of regulatory fines. The regulator aims to control the probability of the firm terminating production, by imposing a closure fine which is as low as possible, while achieving the required reduction in probability. Our method determines the level of fine which establishes a Nash equilibrium in these nonzero-sum games, under uncertainty.
\end{abstract}

Keywords: Regulation, Real Options, Uncertainty, Optimal Control, Probabilistic Constraints

\section{Introduction}

In highly controlled markets such as the public utility industries, the regulator of an industry may impose direct control on the operating strategy of a firm, even though the company operates under exogenous uncertainty (Roseta-Palma and Xepapadeas, 2004). As an example, the UK water regulator OFWAT restricts the number of times a privatized water utility operator can impose a domestic hosepipe ban (Arnell, 1998) - despite uncertainty over the level and pattern of rainfall, and the fact that additional bans may be optimal for the operator and also the user. In other markets, direct regulatory control is not always present or undesirable. A particular form of this concerns the security of supply of many publicly awarded contracts, where the regulator wishes to limit the likelihood that the firm terminates the contract early. One such example is vaccine production in the US, where demand levels and costs can be extremely uncertain (Danzon et al., 2005). Indeed, security of vaccine supply is highly important, since the effect on public health of vaccine shortages can be significant (Helms et al., 2005): during the period 2000 to 2005, approximately one-third of all childhood vaccine shortages in the US are estimated to have been caused by vaccine manufacturers deciding to cease production due to unfavorable economic conditions (Hinman et al., 2006). However, a regulator can exert indirect influence upon the likelihood of a company ceasing to deliver upon a contract, by changing a firms economic parameters (Sappington, 2005; Laffont and Martimort, 2002) and in particular, by the use of financial incentives such as penalty fines (Helms et al., 2005).

Motivated by such examples, this paper presents a quantitative method to solve a model of a regulated industry, where both the operator and regulator have distinct objectives. Our methodology is based upon three core economic assumptions: firstly, that the future profits of a firm depend on the values taken by an exogenous stochastic process, such as a commodity price, level of demand or labor market costs; secondly, that the industry under review has a regulator, who wishes to reduce the probability of early contract termination, by appropriately controlling the size of a fine (or tax) for doing so; and thirdly, that firms follow operating policies which maximize the expected future profit from their operation, taking account of fines (or taxes). Under these assumptions we show how to derive partial differential equations which characterize this termination probability and we describe fast and accurate 
numerical algorithms to determine the optimal level of fine.

We shall assume that the target level for the probability of abandonment is given, although we do not specify how this target level should be set; this level is often a political decision (Holt, 2005) and is clearly outside the scope of this paper. In addition, it is important to note that it is without loss of generality that we consider a penalty fine, as the methods we propose could just as well be used to determine an optimally imposed level of continuously-paid taxation by the regulator. As such, the principles of this paper are broad in its potential areas of application (essentially any regulated industry subject to probabilistic constraints). To highlight this broadness, as well as considering the vaccine supply problem, we also analyze the early termination of a regime-switching mining operation in Section 4: The decision to abandon a large mining project can have significant undesirable implications for the surrounding environment and economy (Evatt et al., 2012; Otto, 2010; Veiga et al., 2001) and a mining industry regulator may therefore seek to reduce the probability of abandonment to a target level.

In a non-public policy context, some business-to-business contracts already incorporate an early termination fee (Bates and Lemmon, 2003), whose primary objective is financial (Sharp et al., 2008). This fee can mitigate any additional financial costs associated with early termination, as it makes the counterparty less inclined to terminate due to the higher cost (Williamson, 1985). One might therefore view this paper as a translation of that general principle into a public policy context. Yet because the two participants within our public policy type problem have different objectives (one monetary, one probabilistic), the mathematical structure is (to the best our of knowledge) novel and sharply distinct to situations in which the two participants are both (valuation maximizing) firms.

The problem of controlling event probabilities in regulated industries has been considered previously in related contexts. In financial regulation, the Value at Risk measure limits the probability of losses above a given size in a portfolio due to market movements (Duffie and Pan, 1997). It does not, however, aim to address behavioral considerations in the construction of financial portfolios, nor the setting of appropriate regulatory fines. Optimal control with probabilistic constraints has been investigated in non-regulatory contexts, such as control engineering (Kandukuri and Boyd, 2002), mathematical finance (Follmer and Leukert, 1999) and operations research (White, 1974), although these studies have only considered the objectives of a single party. Likewise, elements of the mathematical structure of this paper could also be used to solve optimal control problems involving just one participant, who is concerned with the question: how does a firm adjust its operating policy so as to reduce the abandonment probability to a desired level? However, the overriding thrust of this paper considers the strategies of two participants: the firm must choose an operating strategy which maximizes returns, while the regulator chooses the level of a fine, taking account of the firm's operating strategy, in order to match the abandonment probability to the target level. The solutions presented in this paper are therefore Nash equilibria for a nonzero-sum game (Starr and Ho, 1969). In addition, the study of decision probabilities in their own right is a relatively young and expanding area of research within quantitative finance, whose non-linear effects are proving insightful to decision makers (Sarkar, 2000; Lund, 2005; Wong, 2007).

The remainder of this paper is organized as follows. Details of the mathematical methods are presented in Section 2. In Section 3 the method is applied to a regulator wishing to control the security of supply of a vaccine. A more complex, data-driven example is presented in Section 4, where a mining industry regulator wishes to reduce the probability of total abandonment of a gold mining project. We conclude our work in Section 5, where we also discuss potential future extensions. 


\section{Optimal Control with Probabilistic Constraints}

In this section we present the mathematical details of the method, in a manner intended to be sufficiently general to be employed in a range of regulatory contexts. The method is a one-dimensional search over the level $K$ of the fine for abandonment, and may be summarized as follows:

1. Find the optimal control strategy for the current value of $K$

2. Find the abandonment probability for this control strategy

3. Check if the regulators criteria is met. If it does not, update $K$ and return to step 1

In considering the firm's optimal strategy, the essential mathematical tool is the Hamilton-JacobiBellman (HJB) equation, which links controlled Itô diffusions and partial differential equations (PDEs). A controlled Itô diffusion in $\mathbb{R}^{n}$ takes the form

$$
d X_{t}=d X_{t}^{u}=b\left(X_{t}, u_{t}\right) d t+\sigma\left(X_{t}, u_{t}\right) d B_{t},
$$

where $b$ is a function taking values in $\mathbb{R}^{n}$ representing an instantaneous drift, $\sigma$ is the instantaneous volatility function, taking values in $\mathbb{R}^{n \times m}$, and $\left(B_{t}\right)_{t \geqslant 0}$ is a Wiener process in $\mathbb{R}^{m}$. In our setting, the process $\left(X_{t}\right)_{t \geqslant 0}$ represents the time evolution of the economic state of a firm, driven by the noise process $B$ which represents random fluctuations in, for example, commodity or labor prices or demand levels. We assume that the firm will abandon production when its economic state ceases to be favorable, which corresponds to the first time at which $X_{t}$ leaves a predetermined set $H$. We denote this abandonment time by $v$. The function $u$ represents the firm's operating strategy (specified for each possible economic state $X_{t}$ ), which is assumed to be fixed at the outset. At each time $t$, the value $u_{t}$ depends on the economic state $X_{t}$ and may only take values in the admissible set $U$. For further background on stochastic optimal control we refer the reader to Øksendal (2003).

Given an operating strategy $u$, we express the firm's future profits using a running profit function $g$, discounted at the rate of interest $r$. Let $T$ be the time at which the operating license expires. In addition to the running profit, the firm also experiences a final cashflow $h\left(X_{V}\right)$; if $v<T$ then the firm has abandoned early, and so $h\left(X_{V}\right)$ includes the fine. We define the performance function $w^{u}$ to be the firm's expected total profit, net of any fine:

$$
w^{u}(x)=E_{x}\left[\int_{0}^{v} e^{-\hat{r} z} g\left(X_{z}, u_{z}\right) d z+e^{-\hat{r} v} h\left(X_{v}\right)\right],
$$

where $E_{x}$ denotes the expected value when $X_{0}=x \in \mathbb{R}^{n}$. We remove the endogenous behavioral uncertainty due to the firm's choice of operating strategy $u$ by assuming that there exists an optimal strategy $u^{*}$ which maximizes the value of the performance function $w^{u}(x)$. This value is thus given by the function $V^{*}$, where

$$
V^{*}(x)=w^{u^{*}}(x) .
$$

The solution for $V^{*}$ is found by solving an HJB equation of the form

$$
\begin{array}{r}
\sup _{v \in U}\{L V(x)+g(x, v)-\hat{r} V(x)\}=0 \quad \text { in } H \\
\lim _{x \rightarrow y} V(x)=-h(y, v) \text { for } y \in \partial H
\end{array}
$$


where

$$
L \equiv \sum_{i, j=1}^{n} a_{i j} \frac{\partial^{2}}{\partial x_{i} \partial x_{j}}+\sum_{i=1}^{n} b_{i} \frac{\partial}{\partial x_{i}}
$$

and $\left[a_{i j}\right]=\frac{1}{2} \sigma \sigma^{\prime}$, and $H$ is the set of all economic states which itself is often part of the solution to the optimal control. A time derivative is indeed present in (2.4), as time forms part of the stochastic process (2.1): $d t=1 . d t+0 . d B$.

In order to study the effect of varying the level of the fine $K$, we specify the following form for $h$ :

$$
h\left(X_{v}, u_{v}\right)=l\left(X_{v}, u_{v}\right)+K I_{v<T}
$$

where $I_{v<T}$ equals 1 if the firm abandons early (ie., if $v<T$ ) and equals 0 otherwise. Note that in equation (2.6), the fine is not inflation-linked; for longer horizons $T$ it may be appropriate to inflationlink the fine, which corresponds to premultiplying $K$ by a factor $e^{\hat{r} v}$. To make the dependence on $K$ explicit, let us write $V^{*}=V^{*}(x, K)$ and $u^{*}=u^{*}(K)$. Our three steps are now:

1. We first solve (2.4) to obtain $V^{*}(x, K)$ and $u^{*}(K)$. This provides the optimal operating strategy $u^{*}(K)$ under the model, given that the fine is set at level $K$.

2. The firm is now assumed to follow this operating strategy and, under this assumption, its economic state $X^{u^{*}(K)}$ is an (uncontrolled) Itô diffusion. As a result the quantity of interest to the regulator, namely the abandonment probability $P(x)=P(x, K)$ for a firm with initial economic state $x$, is then found as described in Øksendal (2003) by solving a form of the Feynman-Kac formula:

$$
\begin{aligned}
L P(x) & =0 \text { in } \quad H \\
\lim _{x \rightarrow y} P(x) & =I_{t_{y}<T} \text { for } \quad y \in \partial H
\end{aligned}
$$

where $t_{y}$ is the value of the time co-ordinate at the point $y$.

3. We now vary $K$ (which in turn varies $u^{*}$ ) until the abandonment probability is reduced to the level $Y$ required by the regulator. Let $K^{*}$ be this optimal fine, so that

$$
P\left(x, K^{*}\right)=Y \text {. }
$$

By construction of this three step process, where absolute deviation from the target is to be avoided, once the condition (2.8) is met we are in a form of nonzero sum Nash equilibrium (Starr and Ho, 1969).

It is worth noting that, depending on the application, the rate of interest $\hat{r}$ above (which is used to find $V$ ) may be different from the one used to solve the Feynman-Kac formula (2.7) (and hence to find $K$ ). If the economic uncertainty is the price process of a traded commodity, then the market prices effectively determine a risk-adjusted rate of interest. In order to avoid the possibility of arbitrage, it is this rate that must be used to calculate $V$. In contrast, when calculating the abandonment probability the regulator is free to leave the probability undiscounted. Alternatively, a bespoke discount rate may be specified for $P$, in order to place greater weight on early abandonment and correspondingly less weight on later abandonment. 


\section{$2.1 \quad$ Feasibility}

The fine $K^{*}$ obtained above is optimal, in the sense that a lower fine would not achieve the desired reduction in abandonment probability and a higher fine would have a negative societal impact by discouraging the purchase of new licenses. However, the optimal fine may be sufficiently high so that no rational firm would buy a new license. The optimal feasible fine, $K_{f}^{*}(x)$, is therefore

$$
K_{f}^{*}(x)=\min \left\{K_{f}(x), K^{*}(x)\right\},
$$

where $K_{f}(x)$ is the maximum fine the firm can afford; further discussion on this is given by Lear and Maxwell (1998), and the practical implementation of fines (such as collecting performance bonds) is discussed by Holt (2005).

\subsection{Notation}

The notation used in this section is consistent with that generally used in probability theory, in the sense of $\emptyset \mathrm{ksendal}$ (2003). Yet as can be seen, there are three different symbols related to the stochastic process: the set of all possible processes $X$, the individual point in the process $X_{t}^{u}$ and the quantity postaveraging $x$. The notation serves a purpose in deriving the theoretical basis. But given its purpose has been satisfied, we now intentionally relax some of the notational rigor in order for an interdisciplinary reader to more easily move their way through the remaining sections. To be consistent with some of the key work on real options theory and quantitative finance (such as Dixit and Pindyck (1994) and Wilmott et al. (1995)) as far as possible we use just the capital letter to denote the stochastic variable.

\section{Improving security of vaccine supply}

In this section, motivated by Hinman et al. (2006) and Helms et al. (2005), we investigate the problem of increasing the security of supply of a vaccine. We employ a simple real options model, in which a firm is contracted to supply vaccines at a fixed quantity and price, and is exposed to both fixed and uncertain input costs. The only control available to the firm is the early termination of the contract, whilst the industry regulator may wish to use a fine to control the probability of termination. Termination fines are commonly used (Bates and Lemmon, 2003), and the method of Section 2 provides a quantitative method for setting the level of fine, taking account of the profit maximizing behavior of the firm.

Our model in this section is the following. The firm must deliver a fixed number $q$ of doses each year for $T$ years, which are to be sold at an agreed sale price of $s_{c}$ per dose. The firm's costs include a fixed amount of $\varepsilon$ per year and a variable amount $S$ which is uncertain and is assumed to follow a geometric Brownian motion

$$
d S=\mu S d t+\sigma S d B
$$

where $\mu$ is the percentage drift and $\sigma$ is the percentage volatility. The firm's control strategy is simply to terminate the contract when $S$ rises to a predetermined level $S^{*}(t)$. This level may be time-dependent (although we will often suppress the time parameter for notational convenience), and takes account of all termination costs. With the notation of Section 2, the firm exerts no control until the termination time $v$, and so the choice of strategy reduces to the choice of a termination surface $S^{*}$. We supply our own plausible parameter values characterizing the uncertainty and where possible, we use parameter values consistent with those given in Hinman et al. (2006):

$$
\begin{gathered}
\mu=2.5 \% \mathrm{yr}^{-1}, \quad \sigma=0.3 \mathrm{yr}^{-1 / 2}, \quad r=2 \% \mathrm{yr}^{-1}, \quad s_{c}=\$ 30 \mathrm{U}^{-1}, \\
T=5 \mathrm{yr}, \quad I=\$ 100 \mathrm{M}, \quad q=5 \mathrm{M} \mathrm{U} \mathrm{yr}^{-1}, \quad \varepsilon=\$ 1.3 \mathrm{M} \mathrm{yr}^{-1}
\end{gathered}
$$


where $I$ is the initial investment and we suppose that the current level of variable input costs is $S=\$ 10$ $\mathrm{U}^{-1}$, where $U$ refers to a single dose. We suppose that the regulator's target is to reduce the termination probability by $20 \%$ relative to the baseline level when there is no fine, that is:

$$
P\left(v<T \mid K=K^{*}\right)=Y=\frac{4 P(v<T \mid K=0)}{5}
$$

\subsection{Regulator problem: Termination probability}

In the notation of Section 2 we have $n=2$ and $m=1$. Since the firm exerts no control before the termination time, we do not model a control variable $u_{t}$ and (2.1) reduces to

$$
X_{t}=\left[\begin{array}{c}
S_{t} \\
t
\end{array}\right], b\left(X_{t}, u_{t}\right)=\left[\begin{array}{c}
\mu S \\
1
\end{array}\right], \sigma\left(X_{t}\right)=\left[\begin{array}{c}
\sigma S \\
0
\end{array}\right],
$$

where the economic state $X_{t}$ takes values in the region $H$ bounded above (in its first coordinate) by the surface $S^{*}$. Inserting this in equation (2.7), we have the equation governing the probability of contract termination:

$$
\begin{gathered}
\frac{\partial P}{\partial t}+\frac{1}{2} \sigma^{2} S^{2} \frac{\partial^{2} P}{\partial S^{2}}+\mu S \frac{\partial P}{\partial S}=0, \\
P=0 \quad \text { when } t=T, \\
P=1 \quad \text { on } \quad S=S^{*}, \\
P \rightarrow 0 \quad \text { as } \quad S \rightarrow 0 .
\end{gathered}
$$

The solution to this equation depends on the firm's control strategy, which is given by the termination surface $S^{*}$ obtained in Section (3.2) below.

We note that if the contract is held in perpetuity, and if the final cashflow $h$ is constant in time, we obtain a time-stationary version of the problem. These assumptions are commonly used in real options studies (Dixit and Pindyck, 1994), and make closed form solutions available by removing the time dimension from the problem. However, in the setting of this paper, the effect of discounting a constant fine reduces its effect so greatly that such perpetual solutions are uninformative. In the following we therefore explore solutions to finite time horizon problems, using robust numerical algorithms.

\subsection{Operator problem: termination surface}

We now obtain the optimal termination surface $S^{*}$, as a function of the fine level $K$. The surface $S^{*}(K)$ is both an input to the calculation in section (3.1), and the optimal operating strategy for the firm under this model. The firm's running profit is modeled as the sum of the sales, variable costs and fixed costs:

$$
g(S)=q\left(s_{c}-S\right)-\varepsilon
$$

and the function $l$ from (2.6) in section 2.4 represents the capital cost of closure, $C$. Inserting the price process (3.1) and cashflows (3.6) into (2.4), we can write the equation governing the valuation as

$$
\frac{\partial V}{\partial t}+\frac{1}{2} \sigma^{2} S^{2} \frac{\partial^{2} V}{\partial S^{2}}+\mu S \frac{\partial V}{\partial S}-r V+d\left(s_{c}-S\right)-\varepsilon=0
$$




$$
\begin{gathered}
V=0 \quad \text { when } \quad t=T, \\
V=\frac{q s_{c}-\varepsilon}{r}\left(1-e^{-r(T-t)}\right) \quad \text { when } \quad S=0, \\
V=-C-K \quad \text { on } \quad S=S^{*} .
\end{gathered}
$$

This particular equation is a form of HJB equation with 'bang-bang' control. The reason it is written in the same form as the Feynman-Kac equation, is due to the fact the running profit function $g$ is linear and a continuous interval $[a, b]$ of choices is available for $U$; although the HJB equation holds, in this instance it simplifies to solving Feynman-Kac equations on overlapping domains with free boundaries (Øksendal, 2003). However, when the running profit is nonlinear this is no longer true, and the full HJB equation must be solved.

To maximize $V$, the firm must choose the surface $S^{*}$ optimally. This may be achieved using the smooth pasting principle as fully detailed by Dixit (1991). In the case of this abandonment problem, in which there are no future cash flows once abandonment has been completed, the condition is given by,

$$
\frac{\partial V}{\partial S}=0 \quad \text { on } \quad S=S^{*}
$$

\subsection{Numerical Approach}

The boundary $S^{*}(K)$ found in Section (3.2) may now be input to the calculation in Section (3.1), to obtain the abandonment probability. We denote this probability $P(K, S, t)$, again to indicate dependence on $K$. Given the current variable production cost $S$, it is now a matter of a one-dimensional search to find the minimum value of $K$ such that $P(K, S, t)=Y$.

The precise numerical scheme we shall use to solve for the operational control determined via equation (3.7), is a projected successive over relaxation (PSOR) method, which is an accurate procedure for solving such free boundary problems in quantitative finance. The solution to the regulators problem for determining the probability, (3.5), does not involve determining a free boundary, as this boundary is purely an input. As such, one can use a standard implicit finite-difference scheme. Both of these schemes are explained and detailed in Wilmott et al. (1995), where uniqueness has recently been shown to be ensured (Briani et al., 2011).

\subsection{Results}

The solutions to (3.7) and (3.5), when using the parameters given by (3.2), are shown in Figure 1. The top graph shows how the optimal net present value (NPV $=V-I$ ) of the operation is dependent upon the termination fine $K$, and the lower graph shows how the resulting probability of termination varies. The objective of the regulator is to reduce the probability of early termination by $20 \%$, and the fine which achieves this aim is indicated by the intersecting dashed lines in the lower graph: $K^{*}=$ $\$ 5.6 \mathrm{M}$. The introduction of this fine leads to a reduction of the firm's NPV from $\$ 137.5 \mathrm{M}$ to $\$ 136.82 \mathrm{M}$, a reduction in NPV of just $0.5 \%$. As one would expect, the approximate size of this reduction in valuation is given by the probability of abandonment multiplied by the size of the optimal penalty fine.

Figure 2 illustrates the effect of the optimal fine $K^{*}$ on the optimal operating strategy of the firm, where the termination boundaries $S(0)$ (dashed line) and $S\left(K^{*}\right)$ (solid line) are shown. The difference between these two termination prices becomes greater close to contract expiry. It is clear from Figure 

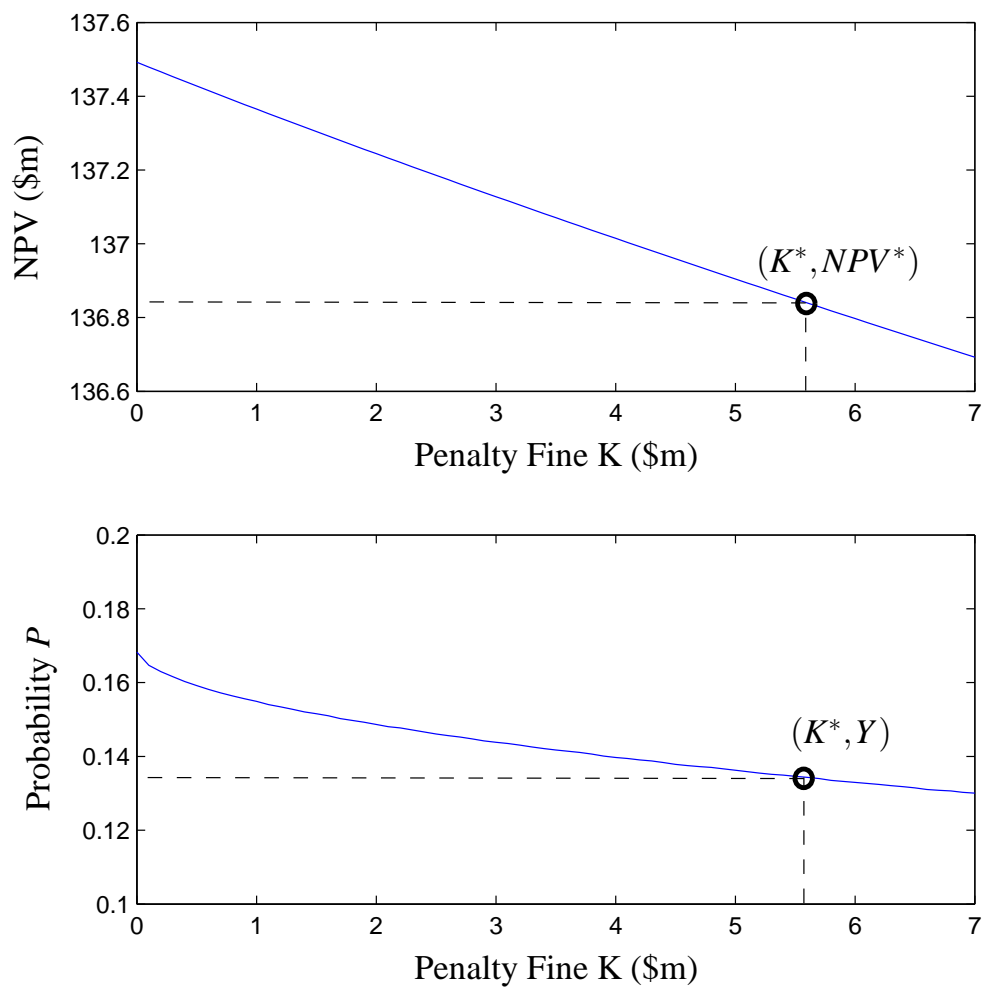

FIG. 1. The top graph shows the firm's NPV for each termination fine $K$, and the lower graph shows the firm's probability of early termination (the quantity of interest to the regulator) for each $K$. The fine $K^{*}$ achieving a $20 \%$ reduction in termination probability is shown in the lower graph (where the curve meets the horizontal dashed line), and the corresponding NPV is given in the top graph

2 that the reduction in termination probability is principally achieved by excluding termination decisions close to expiry of the operating license: this feature also explains the relatively low impact of the termination fine on the firm's NPV.

In Figure 3 we investigate the sensitivity of the results to the parameter values specified in (3.1). The top graph shows how, given the initial price level, the probability of early termination increases as the percentage volatility $\sigma$ is increased. In the bottom graph we show how the probability of termination increases as the percentage drift $\mu$ increases. We note that the main effect is a translation of the curve, so that the objective of a $20 \%$ reduction in termination probability appears robust to uncertainty over the parameters $\mu$ and $\sigma$.

\section{Increasing the societal benefit of an extraction project}

In this section we consider the use of fines to reduce the probability that a mining project will be abandoned early. The societal and economic benefits from a mining project can be large, with increased investment in an area providing increased levels of employment and improved community resources. 


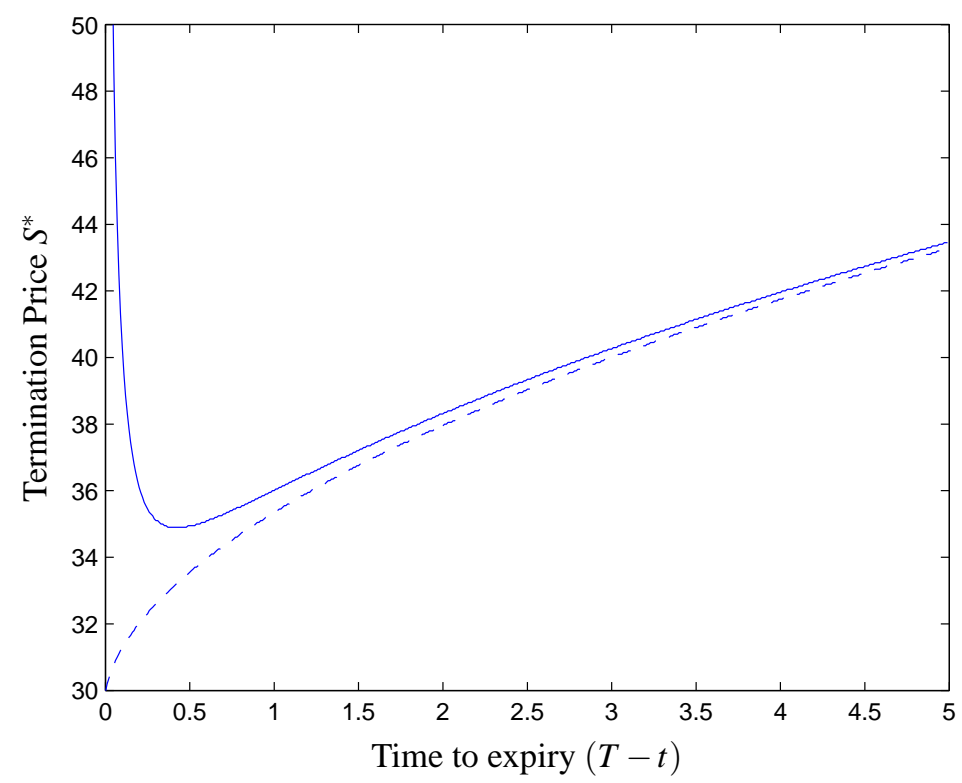

FIG. 2. Optimal termination prices for the manufacturer, viewed as the time to expiry is approached, for two different penalty fines. The continuous line is when the optimal termination fine is imposed upon the operator $K^{*}=\$ 5.6 \mathrm{M}$, and the dashed line shows the optimal strategy when no penalty fine is imposed $K=\$ 0$

When extraction ceases, these benefits also cease, to the detriment of the community (Andrews-Speed et al., 2005); the abandonment of the extraction site may also carry an environmental cost. A recent World Bank report (Otto, 2010) discusses a legal framework within which local governments can ensure more sustainable resource extraction projects. Such considerations include making provisions for new forms of economic activity and employment post-extraction (Veiga et al., 2001). However, these provisions may not address the risk of early abandonment of the mining project due to unfavorable commodity prices. In the context of a regulated market, a suitable equilibrium must be sought between the regulator and mining firm (Kniesner and Leeth, 2004; Otto, 1997). If the equilibrium is achieved through a fine for abandonment, the regulator must avoid deterring this often vital corporate investment (Otto, 2010). We now show that this problem falls within the scope of Section 2, when the exogenous uncertainty $S$ is the commodity price and the operating strategy $u$ is the rate at which the commodity is extracted.

\subsection{Model: the mining operation}

In this example we use empirical data on the ore-grade quality $G$ (grammes of gold per tonne of earth) from a real gold mining operation, whose data has been supplied by Gemcom Software International (a large mining solutions provider). The data is plotted in Figure 4, following an extraction schedule which is scheduled to last 4.9 years at constant extraction rate. In our model, the economic state of the mine is $(S, t, Q)$, where $Q$ is the volume of ore remaining in the mine. We assume that $S$ follows a 

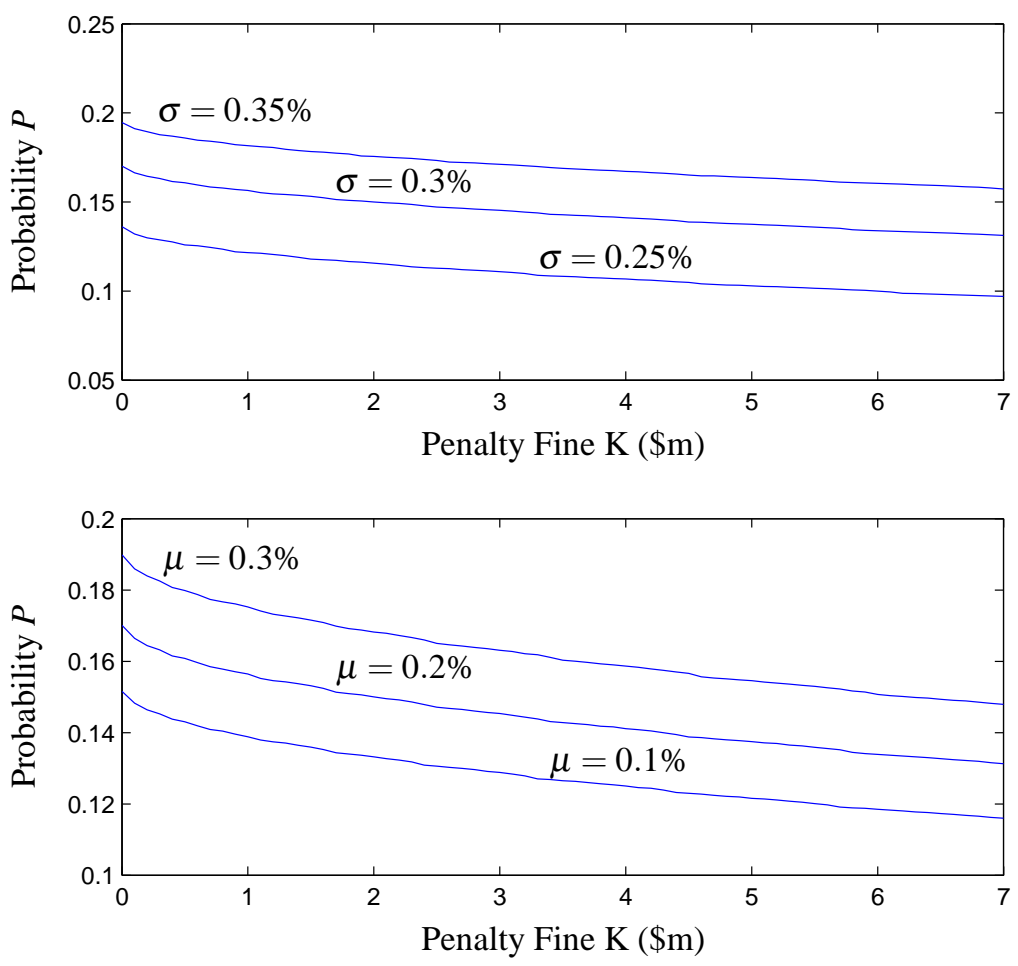

FIG. 3. The sensitivity of the probability of early termination, as one varies the parameters of the stochastic process (3.1). The top graph shows the sensitivity towards the volatility: $\sigma=0.25,0.3$ and $0.35 \%$. The bottom graph shows the sensitivity towards the drift: $\mu=0.1,0.2$ and $0.3 \%$. Unless being varied in the graph, all other parameters are given by (3.2)

mean-reverting Cox-Ingersoll-Ross process, with stochastic dynamics

$$
d S=\kappa(\mu-S) d t+\sigma \sqrt{S} d B
$$

where $\mu$ is the long-term average price of gold and $\kappa$ is the speed of mean reversion. The diffusion $(S, t, Q)$ is controlled by choosing the rate of extraction $q=-\frac{d Q}{d t}$. In the notation of Section 2 we have $n=3, m=1$ and

$$
u_{t}=q_{t}, X_{t}=\left[\begin{array}{c}
S_{t} \\
t \\
Q_{t},
\end{array}\right], b\left(X_{t}, u_{t}\right)=\left[\begin{array}{c}
\kappa(\mu-S) \\
1 \\
-q_{t}
\end{array}\right], \sigma\left(X_{t}, u_{t}\right)=\left[\begin{array}{c}
\sigma \sqrt{S} \\
0 \\
0
\end{array}\right] \text {. }
$$

We take a simple model in which $q$ may have either the value $q_{1}$ or $q_{2}$, with $q_{1}<q_{2}$; we will refer to state 1 as 'normal operation', and to state 2 as 'expanded operation'. As such, this example may be considered a particular form of regime switching diffusion (Chen and Insley, 2012). The capital cost of switching from state 1 to state 2 is $C_{e}$, and switching from state 2 to state 1 is not possible. The mine may be abandoned from either state, incurring a capital $\operatorname{cost} C_{1 a}$ and $C_{2 a}$ from states 1 and 2 respectively. 


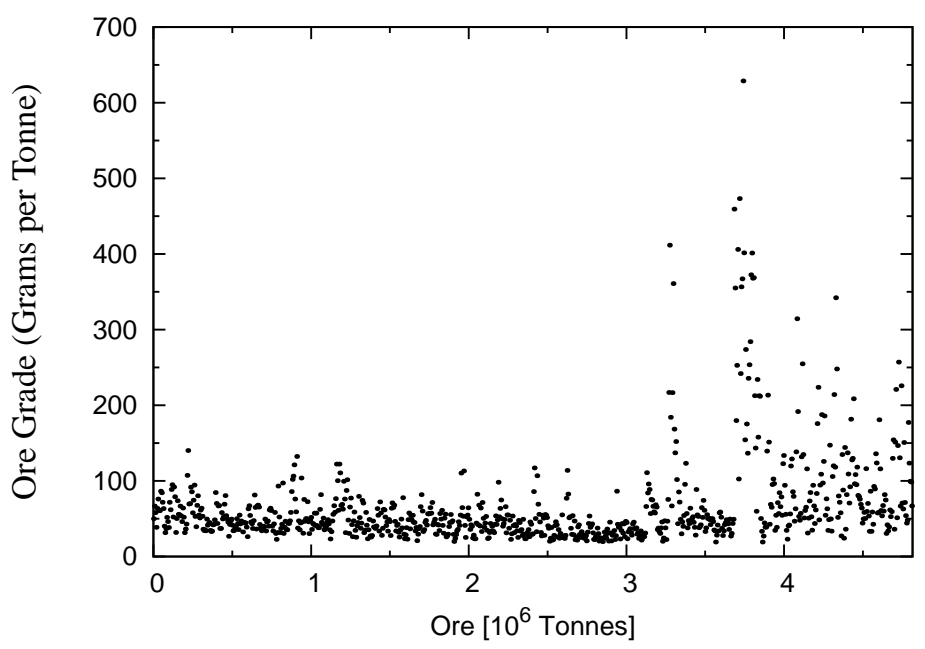

FIG. 4. The ore grade of a gold mine, ordered in a chronological sequence of extraction. The grade, $G$, is measured in grammes per tonne, and the x-axis denotes the amount of remaining ore, $Q$. This data was supplied by Gemcom Software International

The firm's running cost per unit time in state $i$ is $\varepsilon_{i}$, so that the running cashflows for the mine are

$$
g_{i}(S, t, Q)=q_{i} G(Q) S-\varepsilon_{i} .
$$

The operational strategy of any firm is assumed to consist of three price thresholds $S_{e}^{*}(t, Q), S_{1 a}^{*}(t, Q)$ and $S_{2 a}^{*}(t, Q)$, all of which may depend on both the time $t$ and the remaining quantity of ore $Q$. The threshold $S_{e}^{*}(t, Q)$ is the gold price at which the operation expands from state 1 to state 2, while $S_{1 a}^{*}(t, Q)$ and $S_{2 a}^{*}(t, Q)$ are the gold prices at which the project is abandoned, respectively from states 1 and 2 .

The objective of the regulator is assumed to be the introduction of a fine for abandonment which halves the probability of abandonment (so as to improve the security of supply of economic benefit to the surrounding community), relative to the probability without a fine. The remaining parameter values of this gold mine extraction project are given by

$$
\begin{gathered}
\mu=\$ 24.4 \mathrm{gr}^{-} 1, \quad \sigma=25 \%, \quad \kappa=0.01 y r^{-1}, \quad r=8 \% y r^{-1}, \\
I=\$ 50 M, \quad q_{1}=1 M t y r^{-1}, \quad q_{2}=2 M t y r^{-1}, \quad \varepsilon_{1}=\varepsilon_{2}=\$ y r^{-1}, \\
C_{1 a}=\$ 10 M, \quad C_{2 a}=\$ 10 M, \quad C_{e}=\$ 20 M, \quad S_{0}=\$ 25.7 \mathrm{gr}^{-1} .
\end{gathered}
$$

\subsection{Abandonment probability}

Inserting the controlled diffusion (4.2) into equation (2.7), the probability of abandonment is given by the coupled equations

$$
\begin{gathered}
\frac{1}{2} \sigma^{2} S \frac{\partial^{2} P_{1}}{\partial S^{2}}-\frac{\partial P_{1}}{\partial \tau}-q_{1} \frac{\partial P_{1}}{\partial Q}+\kappa(\mu-S) \frac{\partial P_{1}}{\partial S}=0, \\
P_{1}=1 \quad \text { on } \quad S=S_{1 a}^{*}, \\
P_{1}=0 \quad \text { when } \quad \min \{Q, \tau\}=0, \\
P_{1}=P_{2} \quad \text { on } \quad S=S_{e}^{*},
\end{gathered}
$$


and

$$
\begin{gathered}
\frac{1}{2} \sigma^{2} S \frac{\partial^{2} P_{2}}{\partial S^{2}}-\frac{\partial P_{2}}{\partial \tau}-q_{2} \frac{\partial P_{2}}{\partial Q}+\kappa(\mu-S) \frac{\partial P_{2}}{\partial S}=0, \\
P_{2}=1 \quad \text { on } \quad S=S_{2 a}^{*}, \\
P_{2}=0 \quad \text { when } \quad \min \{Q, \tau\}=0, \\
P_{2} \rightarrow 0 \quad \text { as } \quad S \rightarrow \infty,
\end{gathered}
$$

where $\tau=T-t$. These particular boundary conditions are mathematically analogous to hysteresis problem in physics solved by Freidlin et al. (2000). As in the previous section, the solution to the coupled equations (4.5) - (4.6) depends on the firm's control strategy. We now describe, from the point of view of a firm, the optimal choice for the three surfaces $S_{1 a}^{*}(\tau, Q), S_{2 a}^{*}(\tau, Q)$ and $S_{e}^{*}(\tau, Q)$.

\subsection{Operator}

From the price process (4.1), controlled diffusion (4.2) and running cash flows (4.3), we can insert them into equation (2.4), to obtain the following PDE for the mines expected profit:

$$
\begin{gathered}
\frac{1}{2} \sigma^{2} S \frac{\partial^{2} V_{1}}{\partial S^{2}}-\frac{\partial V_{1}}{\partial \tau}-q_{1} \frac{\partial V_{1}}{\partial Q}+\kappa(\mu-S) \frac{\partial V_{1}}{\partial S}-r V_{1}+q_{1} G S-\varepsilon_{1}=0 \\
V_{1}=0 \quad \text { when } \quad \min \{\tau, Q\}=0 \\
V_{1}=-C_{1 a}-K \quad \text { when } S=S_{1 a}^{*} \\
V_{1}=V_{2}-C_{e} \quad \text { on } S=S_{e}^{*},
\end{gathered}
$$

and

$$
\begin{gathered}
\frac{1}{2} \sigma^{2} S \frac{\partial^{2} V_{2}}{\partial S^{2}}-\frac{\partial V_{2}}{\partial \tau}-q_{2} \frac{\partial V_{2}}{\partial Q}+\kappa(\mu-S) \frac{\partial V_{2}}{\partial S}-r V_{2}+q_{2} G S-\varepsilon_{2}=0, \\
V_{2}=0 \quad \text { when } \quad \min \{\tau, Q\}=0 \\
V_{2}=-C_{2 a}-K \quad \text { when } \quad S=S_{2 a}^{*} \\
V_{2} \sim S \quad \text { as } \quad S \rightarrow \infty .
\end{gathered}
$$

We refer to Brennan and Schwartz (1985) for the justification of these boundary conditions. In addition, for optimality to be obtained, we require that at each of the transitions the smooth pasting condition must hold:

$$
\frac{\partial V}{\partial S}=0 \quad \text { on } \quad S=\left\{S_{e}^{*}, S_{1 a}^{*}, S_{2 a}^{*}\right\} .
$$

The boundaries $S_{1 a}^{*}(\tau, Q), S_{2 a}^{*}(\tau, Q)$ and $S_{e}^{*}(\tau, Q)$, which again depend on $K$ (although we have suppressed this for notational convenience), may be obtained from (4.7)—(4.8) via a PSOR numerical method and input to the calculation in section (4.2). A one-dimensional search is again sufficient to obtain the minimum value of $K$ achieving the desired reduction in abandonment probability under the model. 

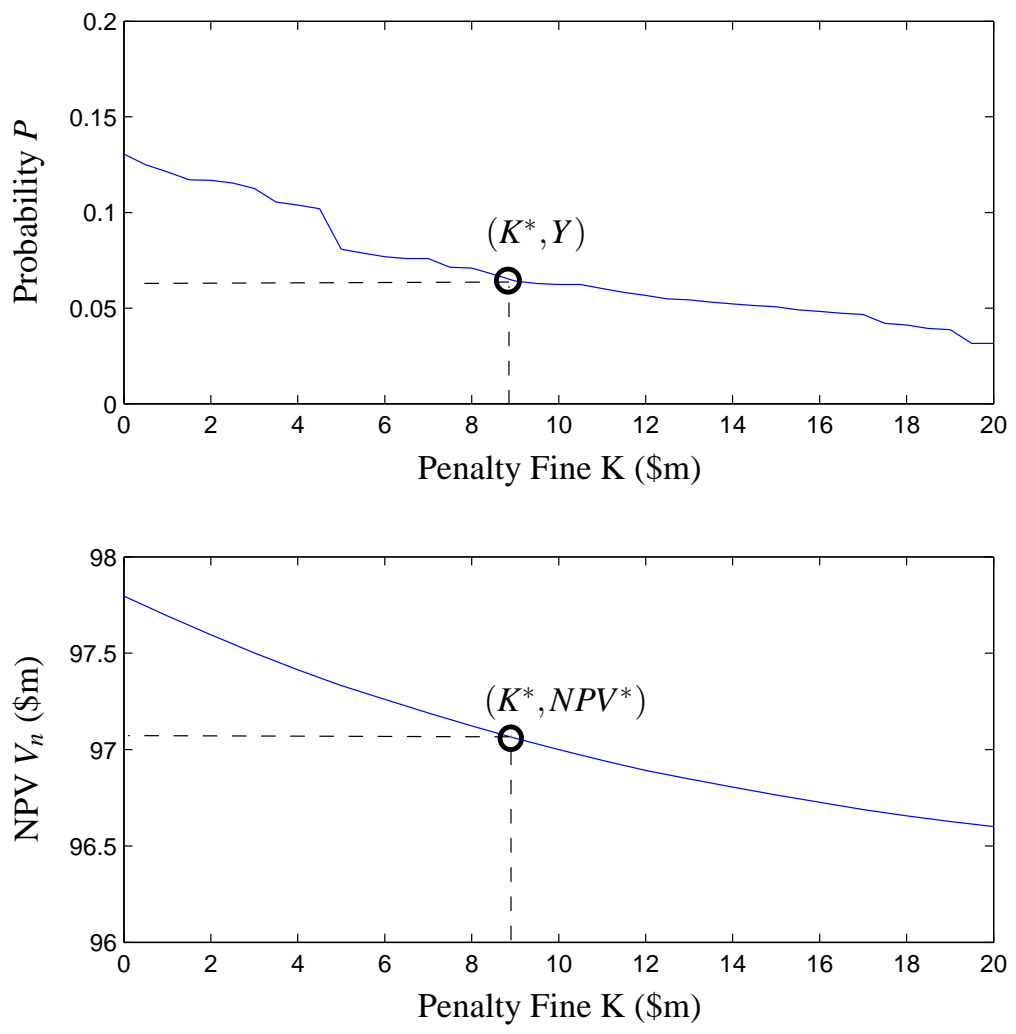

FIG. 5. The probability of abandonment (top graph) and NPV (bottom graph) of a gold mining project, plotted as a function of abandonment penalty fine. The dashed lines show where the regulators objective is met - which is to half the probability of abandonment - and the resulting optimal NPV. The results are plotted at $\$ 0.5 M$ intervals, using the parameters values of (4.4)

\subsection{Results}

Since equations (4.7)—(4.8) are convection dominated in the $Q$ variable, we obtain numerical results by the semi-Lagrangian method, as utilized by Evatt et al. (2011) and Chen and Forsyth (2007). In Figure 5 we show the probability of abandonment versus level of fine $K$ (top graph), and the mining project's NPV versus $K$ (bottom graph). The optimal level for $K$ is $K^{*}=\$ 8.85 \mathrm{M}$ with an associated NPV of $\$ 97.05 \mathrm{M}$, which compares to $\$ 97.8 \mathrm{M}$ when $K=0$. The halving of abandonment probability is thus achieved in return for a reduction in NPV of $\$ 0.75 \mathrm{M}$, or $0.8 \%$. The roughness of Figure 5 (top) is a consequence of roughness in the ore grade data as shown in Figure 4. Figure 6 shows the optimal thresholds for changing operational state, when no fine is imposed (top) and when the optimal fine $K=\$ 8.85 M$ is imposed (bottom). The higher dashed line is the optimal price to expand operation $S_{e}^{*}$, the lower dotted line is the optimal price to abandon from the expanded state $S_{a 2}^{*}$ and the continuous line is the decision to abandon from normal operation $S_{a 1}^{*}$. The most significant difference between the two operating strategies is found towards the end of extraction, as $Q$ approaches 0 . 

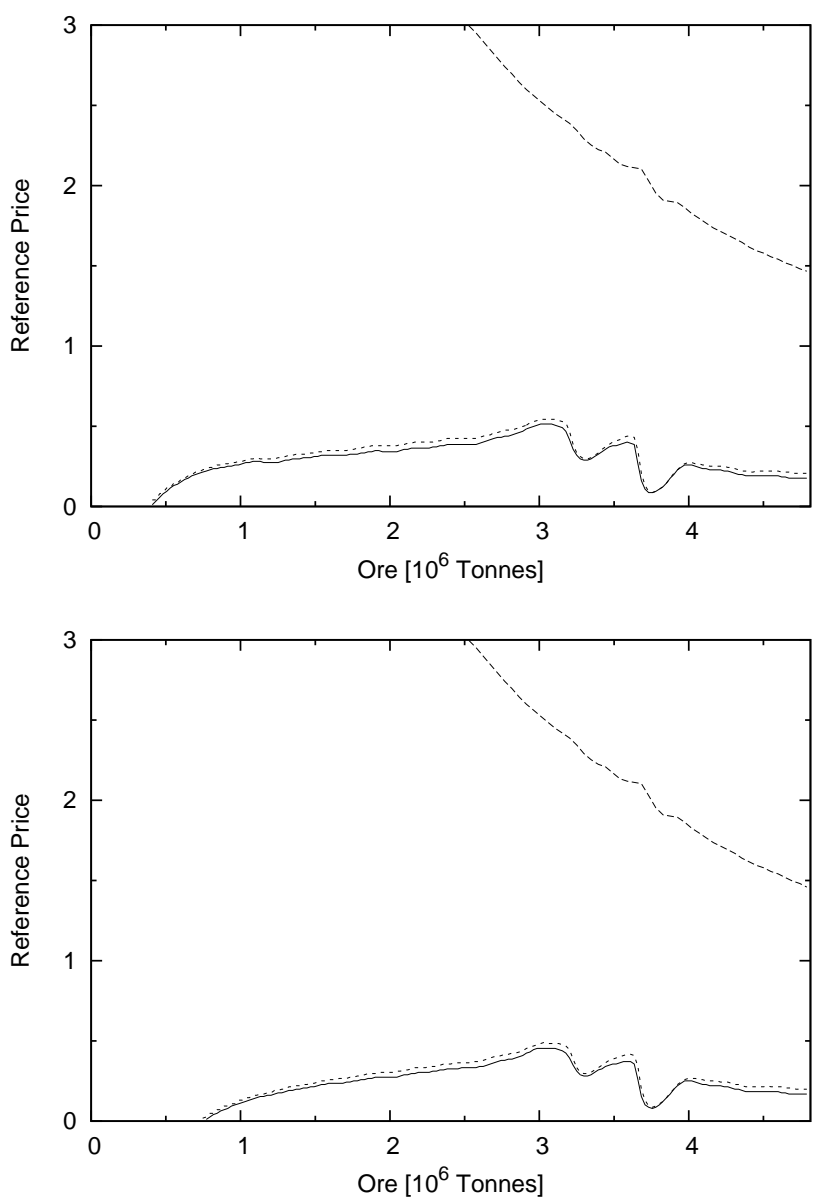

FIG. 6. Graphs showing the optimal prices for when to change operational state of a gold mine, for when no penalty fine is present (top graph) and for when the optimal penalty fine, $K=\$ 8.85 \mathrm{M}$, is imposed (bottom graph). In each figure, results are shown for the optimal expansion decision $S_{e}^{*}$ (higher dashed line), the optimal abandonment decision from the expanded state $S_{a 2}^{*}$ (lower dashed line), and the optimal abandonment decision from the normal extraction state $S_{a 1}^{*}$ (lower continuous line). The graphs were calculated using the parameters values of (4.4)

\section{Conclusion and discussion}

We have presented a method for determining regulatory fines, payable upon contract termination, which establish a Nash equilibrium between an industry regulator and a firm, in the presence of exogenous uncertainty: the regulator aims to increase the security of contract delivery, whilst the firm aims to maximise its valuation. The method uses the partial differential equations which govern both the firm's expected profit and the values of regulatory interest. It therefore contrasts with scenario analysis (Postma and Liebl, 2005) by considering all possible future scenarios, and is exact in that it does not require simulations.

Our work is applicable whenever the evolution of a firm's economic state can be modeled by a 
controlled Itô diffusion, and its profits can be expressed in the form (2.2). We have given illustrative applications to two example regulatory scenarios. However, if the uncertainty in question exhibited jumps -and was therefore a form of Lévy process- one could still make use of the principles and majority of mathematics within this paper. This potential model extension would introduce integral terms with the underpinning differential equations, turning them into forms of partial integral differential equations (PIDEs), whose solutions can be extraction via numerical schemes such as that suggested in Thompson et al. (2004).

Extensions of this work can be made, and need not be restricted to the early termination of contracts. This is because many other firm decisions can attract a regulators attention, who may then seek to utilise penalty fines in order to reduce their likelihood. For an interesting example within financial services regulation in which (non-controlled) regulatory fines are imposed on profit-maximizing banks within a Basal II setting, see Jimenez-Martin et al. (2009).

\section{Funding}

This work was supported by the Engineering and Physical Sciences Research Council (EPSRC) UK, via the University of Manchester Knowledge Transfer Account.

\section{Acknowledgements}

We are grateful to both EPSRC for funding this project, and Gemcom Software International, who provided us with data and consultation regarding the running of mining operations.

\section{References}

Andrews-Speed, P., Ma, G., Shao, B., Liao, C., 2005. Economic responses to the closure of small-scale coal mines in chongqing, china. Resources Policy 30 (1), 39 - 54.

Arnell, N. W., 1998. Climate change and water resources in britain. Climatic Change 39, 83-110, 10.1023/A:1005339412565.

Bates, T. W., Lemmon, M. L., 2003. Breaking up is hard to do? an analysis of termination fee provisions and merger outcomes. Journal of Financial Economics 69 (3), 469 - 504.

Brennan, M. J., Schwartz, E., 1985. Evaluating natural resource investments. The Journal of Business 58 (2), 135-157.

Briani, A., Camilli, F., Zidani, H., 2011. Approximation Schemes for Monotone Systems of Nonlinear Second Order Partial Differential Equations: Convergence Result and Error Estimate, to appear in Differential Equations and Applications.

Chen, S., Insley, M., 2012. Regime switching in stochastic models of commodity prices: An application to an optimal tree harvesting problem. Journal of Economic Dynamics and Control 36 (2), 201 - 219.

Chen, Z., Forsyth, P., December 2007. A semi-lagrangian approach for natural gas storage valuation and optimal operation. SIAM J. Sci. Comput. 30, 339-368.

Danzon, P. M., Pereira, N. S., Tejwani, S. S., 2005. Vaccine supply: A cross-national perspective. Health Affairs 24 (3), 706-717. 
Dixit, A., 1991. A simplified treatment of the theory of optimal regulation of brownian motion. Journal of Economic Dynamics and Control 15 (4), 657 - 673.

Dixit, A., Pindyck, R., 1994. Investment under Uncertainty. Princeton University Press.

Duffie, D., Pan, J., 1997. An overview of value at risk. The Journal of Derivatives 4 (3), 7-49.

Evatt, G. W., Johnson, P., Duck, P., Howell, S., Moriarty, J., 2011. The expected lifetime of an extraction project. Proceedings of the Royal Society A: Mathematical, Physical and Engineering Sciences 467, 244-263.

Evatt, G. W., Soltan, M. O., Johnson, P. V., 2012. Mineral reserves under price uncertainty. Resources Policy 37 (3), 340 - 345.

Follmer, H., Leukert, P., 1999. Quantile hedging. Finance and Stochastics 3, 251-273.

Freidlin, M. I., Mayergoyz, I., Pfeiffer, R., Aug 2000. Noise in hysteretic systems and stochastic processes on graphs. Physics Review E 62 (2), 1850-1855.

Helms, C. M., Guerra, F. A., Klein, J. O., Schaffner, W., Arvin, A. M., Peter, G., 2005. Strengthening the nations influenza vaccination system: A national vaccine advisory committee assessment. American journal of preventive medicine 29, 221-226.

Hinman, A. R., Orenstein, W. A., Santoli, J. M., Rodewald, L. E., Cochi, S. L., 2006. Vaccine shortages: History, impact, and prospects for the future*. Annual Review of Public Health 27 (1), 235-259.

Holt, L., 2005. Utility service quality-telecommunications, electricity, water. Utilities Policy 13 (3), 189 - 200, special Issue on Utility Service Quality.

Jimenez-Martin, J.-A., McAleer, M., Perez-Amaral, T., 2009. The ten commandments for managing value at risk under the basel ii accord. Journal of Economic Surveys 23 (5), 850-855.

Kandukuri, S., Boyd, S., 2002. Optimal power control in interference-limited fading wireless channels with outage-probability specifications. Wireless Communications, IEEE Transactions on 1 (1), 4655 .

Kniesner, T. J., Leeth, J. D., 2004. Data mining mining data: Msha enforcement efforts, underground coal mine safety, and new health policy implications. Journal of Risk and Uncertainty 29, 83-111.

Laffont, J., Martimort, D., 2002. The Theory of Incentives. Princeton University Press.

Lear, K. K., Maxwell, J. W., 1998. The impact of industry structure and penalty policies on incentives for compliance and regulatory enforcement. Journal of Regulatory Economics 14, 127-148, 10.1023/A:1008005201435.

Lund, D., 2005. How to analyze the investmentuncertainty relationship in real option models? Review of Financial Economics 14 (3-4), 311 - 322.

Øksendal, B., 2003. Stochastic differential equations: an introduction with applications. Universitext (1979). Springer.

Otto, J. M., 1997. A national mineral policy as a regulatory tool. Resources Policy 23 (1-2), 1 - 7 . 
Otto, J. M., 2010. Community development agreement: Model regulations and example guidelines. World Bank Report, 61482 1, 1 - 84.

Postma, T. J. B. M., Liebl, F., 2005. How to improve scenario analysis as a strategic management tool? Technological Forecasting and Social Change 72 (2), $161-173$.

Roseta-Palma, C., Xepapadeas, A., 2004. Robust control in water management. Journal of Risk and Uncertainty 29, 21-34.

Sappington, D. E. M., 2005. Regulating service quality: A survey. Journal of Regulatory Economics 27, $123-154$.

Sarkar, S., 2000. On the investmentuncertainty relationship in a real options model. Journal of Economic Dynamics and Control 24 (2), $219-225$.

Sharp, N., Newton, D., Duck, P., 2008. An improved fixed-rate mortgage valuation methodology with interacting prepayment and default options. The Journal of Real Estate Finance and Economics 36, 307-342.

Starr, A. W., Ho, Y. C., 1969. Nonzero-sum differential games. Journal of Optimization Theory and Applications 3, 184-206, 10.1007/BF00929443.

Thompson, M., Davison, M., Rasmussen, H., 2004. Valuation and optimal operation of electric power plants in competitive markets. Operations Research 52 (4), 546-562.

Veiga, M. M., Scoble, M., McAllister, M. L., 2001. Mining with communities. Natural Resources Forum 25 (3), 191-202.

White, D. J., 1974. Dynamic programming and probabilistic constraints. Operations Research 22 (3), pp. 654-664.

Williamson, O. E., 1985. Assessing contract. Journal of Law, Economics, and Organization 1 (1), 177208.

Wilmott, P., Howison, S., Derwynne, J., 1995. The Mathematics of Financial Derivatives: A Student Introduction. Cambridge University Press.

Wong, K., 2007. The effect of uncertainty on investment timing in a real options model. Journal of Economic Dynamics and Control 31 (7), 2152 - 2167. 